\title{
E-SPAN: Enhanced-SPAN with Directional Antenna
}

\author{
Vivek Kumar, Thenmozhi Arunan, N. Balakrishnan \\ Department of Supercomputer Education and Research Centre \\ Indian Institute of Science \\ Bangalore, 560012 INDIA \\ 080-293-2450 \\ vivek@rishi.serc.iisc.emet.in, then@mmsl.serc.iisc.ernet.in, balki@serc.iisc.ernet.in
}

\begin{abstract}
A Mobile Ad-hoc NETwork (MANET), also referred as Independent Basic Service Set (IBSS), has nodes which not only sendlreceive'packets associated with them but also co-operate in forwarding data and control packets for the network. Efficient use of energy by the mobile nodes is very important to increase the lifetime of MANET. Since nodes seldom have packets to receive, they remain in Idle mode for long periods of time which cause a lot of energy drain. Coordination algorithms reduce the time a node is in the Idle mode by keeping it in Sleep mode as much as possible while coordinators buffer packets for these sleeping nodes.
\end{abstract}

For a given transmit energy, directional antenna has larger transmit range as compared to omni-directional antenna. This feature of directional antenna can be used in protocols which assumes a sparse network. We have chosen SPAN [I] to validate our above claim. SPAN is a protocol which elects a sparse network (i.e. backbone) of coordinators to buffer packets for sleeping nodes. Enhanced SPAN (E-SPAN) is the modified SPAN using directional antenna. Simulations prove the effectiveness of directional antenna and show that E-SPAN reduces average power consumed by the nodes in the network by about $20 \%$.

\section{INTRODUCTION}

The network interface is a major cause of energy drain in a mobile node. Hence, optimizing various operations of a network interface to reduce the energy consumed is paramount for increasing the lifetime of MANET. The typical states of a wireless node are Send, Receive, Awake (Idle) and Sleep [4]. Detailed measurements of the energy consumed by these above states show that power utilized in Idle mode is significant $[1,4]$. Nodes spend most of their uptime in Idle mode, sensing the medium if the packets are destined for them. IEEE 802.1I defines a Power Saving Mode (PSM) in which nodes can power down their transceiver and switch to doze or Sleep mode. Power conservation is obtained by minimizing the time a node is in Idle mode and maximizing the time in Sleep mode. In the IEEE 802.1 I PSM mode, the sender should first ensure if the receiver is active before it can send the packets. Stations in an IBSS use Announcement Traffic Indication Map (ATIM) frames to preempt other stations from sleeping. All stations in an IBSS have to be in active state for the period of ATIM window after Beacon transmission in order to receive the ATIM frames. Active stations buffer packets for other nodes in Sleep mode.
Instead, of having random active nodes buffering data for others, a coordination algorithm can be devised which will designatecertain nodes as coordinators while other nodes can be in PSM for that interval of time. SPAN provides such a coordinator election algorithm. The coordinators stay-active and perform multi-hop packet routing, while all the other nodes except the senders and receivers remain in Sleep mode. Each node periodically broadcasts HELLO messages that contain the node's position, node's status (coordinator or not), its current coordinators, and its current neighbors. From the HELLO message each node constructs a list of its neighbors and coordinators and for each neighbor, a list of its neighbors and coordinators. The election process is done in a decentralized manner using just the local information available at the node.

IEEE 802.11 assumes omni-directional transmission for all packets. Collisions resulting from hidden nodes are hard to detect in wireless networks. To eliminate hidden node problem, IEEE 802.1 I uses RTS/CTS. This silences all the nodes in the vicinity of the sender and the receiver while resulting in only a single successful communication. In the case of directional antennas, two pair of nodes located in each other's vicinity can potentially communicate simultaneously. depending on the direction of their transmission. This increases the spatial reuse of the wireless channel [2]. Use of Directional antenna also has the advantages of higher gain and lower interference. Higher gain allows the node to communicate with other nodes in fewer hops and this results in lower latency.

The main aim of this paper is to study the effectiveness of using directional antennas for protocols which form a,sparse network. The advantages offered are evaluated in terms of energy efficiency. In our implementation of E-SPAN. the performance improvement is observed as the reduction in the number of coordinators and reduction in the energy consumed by the network.

\section{RELATED WORK}

In [4], detailed measurements of the energy consumed during send, receive and promiscuous receive have been made. A good observation made is that the energy consumed in the Idle mode is not negligible. Energy conserved is not synonymous with bandwidth utilization. Energy aware protocol design should consider factors such as the relative proportions of broadcast and point-to-point traffic. We have used this ob- 
servation to select the type of antenna for the different type of traffic.

The Directional MAC (D-MAC) [2] protocol suggests the use of directional antennas for spatial reuse. Directional channel reservation is done using Directional RTS (DRTS) and Directional CTS (DCTS) followed by directional DATA and directional ACK. It has the problems of a new form of hidden node and deafness which arise due to unequal gain and shape of the coverage regions of directional and omni-directional antenna. D-MAC has not analyzed the use of directional antennas in IEEE 802.I I PSM. We have modified and validated the use of directional antennas for IEEE 802.1 I PSM. Work in [3] is an extension of [2] which analyzes the effect of directional antenna on the performance of the routing protocol.

In SPAN [I], the connectivity of MANET is maintained using a set of coordinators. The coordinator election is done in a distributed way. SPAN intends to save energy by putting most of the nodes in the Sleep mode. Coordinators buffer the packets for the nodes in Sleep mode. These elected coordinators forms a connected backbone (i.e. a sparse network) for traffic transmission. Directional antenna due to their longer reach is more suitable for sparse networks. We have chosen SPAN to verify our claim. For saving energy in MANET, using directional antenna proves to he supplementary to the approach of SPAN.

In [6] a comparative study of the different MAC protocols that have been implemented to reduce the collisions that occur in Omni transmission and the benefits of directional antennas are listed. Its emphasis is on the evaluation of spatial reuse of the medium access protocols.

\section{PROBLEM FORMULATION}

The algorithms which use cluster-heads or coordinators, reduces the the topology of the nodes communicating in the MANET to a sparse network. Routing protocols uses clusters (e.g.[9]) to reduce'broadcast redundancy, improve QoS and security. Coordination algorithms (e.g. SPAN [1]) use coordinators to make a backbone of Active nodes which buffer the packets for the nodes in Sleep mode. For a given transmit power, since directional antenna has a longer reach, it is well suited for such sparse networks. The focus of this paper is on the use of directional antenna for such sparse networks and energy efficiency is used as the evaluation metric.

SPAN is a coordination algorithm which uses omnidirectional antenna for communication between nodes. We have Enhanced SPAN to utilize directional antennas. Performance analysis of networks running the coordination algorithms SPAN and E-SPAN is done to analyze the effectiveness of directional antennas.

\section{NETWORK MODEL}

Each wireless node has both Omni and Directed beam antenna capability. Same battery power is used to transmit in both directional and omni-directional modes. Our model does not have Transmit Power Control (TPC). Since directional antenna has greater gain than the Omni antenna, the transmission range of directional beam is greater than omnidirectional beam as shown in Figure 1. Receiver is always in the omni-directional mode while transmitter uses either omni or directional antennas. Only one antenna can he enabled at a time. Switching time between the two modes is considered as negligible.

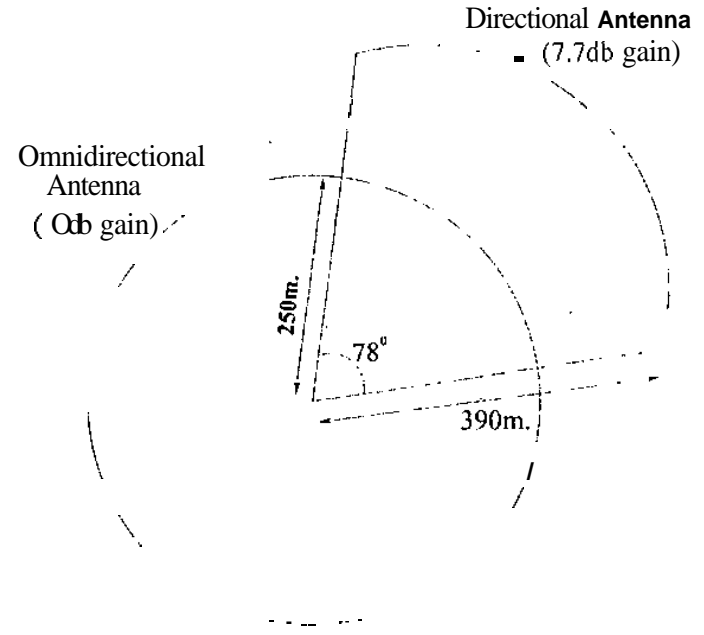

Figure 1. Each node has Omni-directional Antenna and Directional Antenna. Only one antenna can be enabled at a time. Directional antenna has larger range than Omni-directional antenna. The Coverage Area of Directional antenna is less compared to'that of Omni-directional Antenna. The radiation pattem of Directional antenna covers a sector of 78 degrees.

Geographical forwarding protocol is used for routing. Each node knows its location information from Global Positioning System (GPS). For directional transmission of unicast packets. D-MAC needs to know the location information of its neighbors. E-SPAN maintains this information in the neighbor table. E-SPAN sends to D-MAC the transmission profile which contains the location information of the intended receiver node. IEEE 802.11 with PSM is modified to support directional antenna.

\section{MODIFICATIONS TO MAC AND SPAN}

Modifications are done to IEEE 802.11 (PSM) and SPAN in order to use directional antenna. We henceforth refer to the node's neighbors which are in the directional range as directional neighbors. 


\section{Cellular Networks / 677}

IEEE 802.11 Ad hoc Power Saving Mode (PSM)

For IBSS, IEEE 802.1 I provides the option to keep the nodes in PSM. It uses periodic beacons to synchronize nodes in the network. A beacon period starts with ATIM window, during which all nodes are listening and pending traffic transmissions are advertised. A node which receives advertisement of unicast data for itself, acknowledges hack and stays awake for the rest of the beacon period. Otherwise, it can turn itself off at the end of the ATIM window, until the beginning of the next beacon period. After the ATIM window, advertised trafficis transmitted.

\section{Improvements in IEEE 802.11 for Directional Antenna}

In our model we suggest the use of specific type of antenna (omni or directional) based on the packet type. All the broadcast frames are transmitted through omni-directional antenna. While all point-to-point (unicast) frames are transmitted through directional antenna. Henceforth we will use the terms broadcast and omni-directional propagation and unicast and directional propagation interchangeably.

IEEE 802.1 I frames like unicast ATIM, unicast DATA, RTS/CTS and ACK are sent directionally. Now unicast ATIM is also used to advertise'to the nodes which are in the directional reach. Data buffered for the two hop neighbors which are in the directional reach of the node is delivered in a single : transmission. This saves energy by reducing the communication and.buffer overheads. All broadcast packets like beacons, broadcast ATIM, broadcast DATA are sent using omnidirectional antenna.

\section{SPAN details}

SPAN periodically runs election and withdrawal algorithms for making the coordinator backbone. A non-coordinator node becomes a coordinator if it discovers that two of its neighbors cannot reach each other directly or via one or two coordinators. A node withdraws itself as a coordinator if it finds that all pairs of its neighbors can reach each other directly or via a coordinator or two coordinators. The node running election algorithm also takes into account its remaining energy before declaring itself a coordinator. If a node has less energy then it has less probabilitiy to he elected as a coordinator. This ensures rotation of coordinators in the network and hence introduces fairness in energy consumed by the nodes for coordination function.

\section{Improvements in SPAN: E-SPAN}

SPAN DATA is sent in directional mode while HELLO messages from SPAN are sent in omni mode. HELLO packets are modified to include the location information of the neighbor's neighbor. Nodes store this location information in their neighbor table. To transmit a unicast DATA, E-SPAN sends this location information to D-MAC as the transmission profile. D-MAC uses this transmission profile to send unicast data to its neighbor by heamsteering the directional antenna to the direction of its neighbor. Directional RTSICTS (DRTSIDCTS) reserves the channel for the data delivery to directional neighbor. Directional ACK confirms the delivery of data to the directional neighbor.

A node's neighbor's neighbor which is in the direct reach can now be considered as one hop neighbor (or directional neighbor). For this the node calculates the distance between itself and the likely directional neighbor using the location information of the neighbor's neighbor delivered by the neighbor's HELLO packet. The neigbhor table is updated if the neigbhor's neighbor is within the direct reach of the node and is included as its direct neighbor. Nodes also make use of this location information to elect themselves as coordinator using the Improved Coordinator Election Algorithm Now since a elected coordinator serves the directional neighbors as its direct neighbor, the number of coordinators for a given network reduces. This makes the backbone more sparse and thus network using directional antenna results in more energy efficiency.

To use the above enhancements, the Coordinator Election Algorithm is modified. The Improved Coordinator Election A/gorithm is shown below:

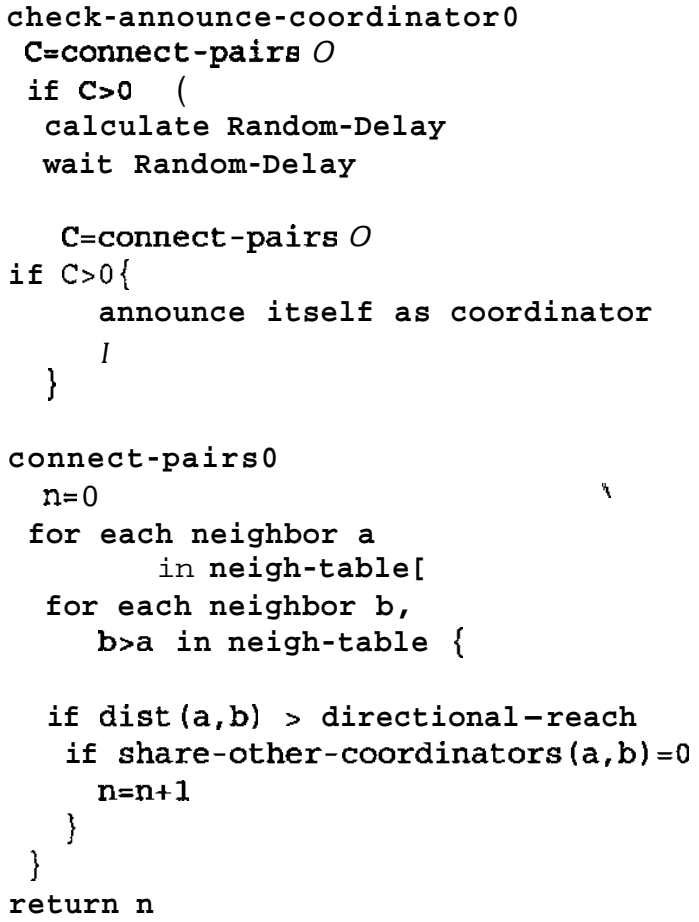

\section{Simulation MOdel}

This section presents the results of E-SPAN implemented with modified IEEE 802.1I (as described in section 5.2) and geographical forwarding routing protocol. We did our simulations in $n s-2$ simulator [8]. To compare the results, most 


\section{TENCON 2003/678}

of the simulation parameters are kept same as in [1]. The simulations are done with 120 nodes for varying area of coverage. Pause time is chosen as 60 seconds. There are $\mathbf{2 0}$ sourceldestination nodes which are placed randomly on a square terrain. Nodes use $2 \mathrm{Mbps}$ bandwidth. Nodes move at 0 to $20 \mathrm{~m} / \mathrm{s}$ with random way-point model. Omni-directional antenna has $250 \mathrm{~m}$ as the transmission range with unity gain. The directional antenna has a gain of $7.7 \mathrm{~dB}$, beamwidth of 78 degrees [5] and range of $391 \mathrm{~m}$. as shown in Figure I. A node transmits using either of the antennas depending upon the frame type while it receives only with omni-directional antenna. Data rate is chosen to be CBR at 3 packet per seconds, each packet is of size 128 , bytes.

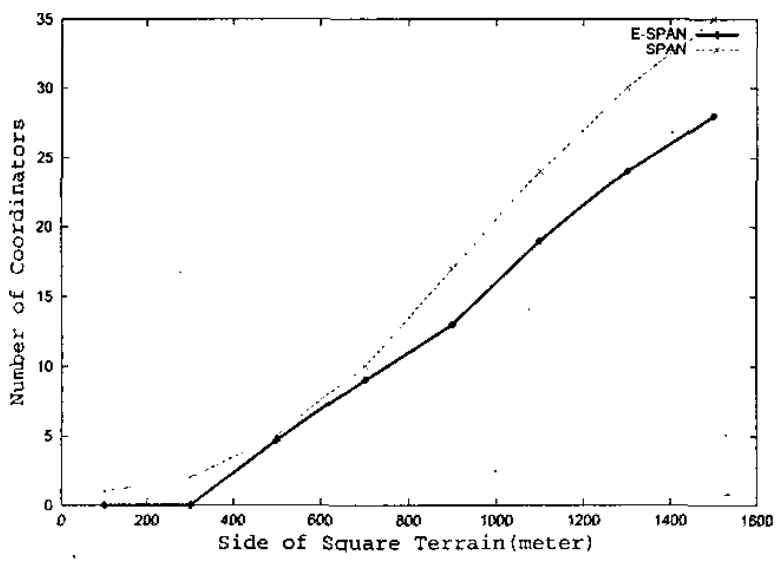

Figure 2. Number of Coordinators Elected vs. Side of Square Terrain

\section{Number of Coordinatorsfor varying Terrain Size}

We have compared the number of coordinators elected in SPAN and E-SPAN for various square terrains. The size of terrain varies from $100 \mathrm{~m} \mathbf{X} 100 \mathrm{~m}$ to $1500 \mathrm{~m}$ X $1500 \mathrm{~m}$. The results presented in figure $\mathbf{2}$ show a significant reduction in the number of elected coordinators when E-SPAN is used. In E-SPAN when the area is small (for the case of $100 \mathrm{~m} \mathrm{X} 100 \mathrm{~m}$ to $300 \mathrm{~m} \times 300 \mathrm{~m}$ ) no coordinator is elected as the nodes send and receive data using IEEE 802.1 I PSM. However SPAN elects 1 coordinator even for a very small network.

When the area is $500 \mathrm{~m} \times 500 \mathrm{~m}$, there is no significant advantage that is offered by E-SPAN, since both E-SPAN and SPAN elect the same number of coordinators. This is due to the fact that the network is still dense and the omni range and largest distance between any two nodes are comparable. However, when the area becomes larger, the advantage of using the directional beam in E-SPAN becomes apparent. from Figure 2, it is seen that the number of coordinators in E-SPAN reduces significantly with increasing area.

\section{Sleep and ldle timefor SPAN and E-SPAN}

The Sleep time and Idle time for both SPAN and E-SPAN are presented in Table 1. The simulation are done for 500 sec-

\begin{tabular}{|l||l|l||l|l||l|l|}
\hline \multicolumn{1}{|c||}{ Area } & \multicolumn{2}{c|}{$500 \mathrm{~m} \times 500 \mathrm{~m}$} & \multicolumn{2}{c||}{$1100 \mathrm{~m} \times 1$ 10Om } & \multicolumn{2}{c|}{$1500 \mathrm{~m} \times 1500 \mathrm{~m}$} \\
\hline \hline & SPAN & ESPAN & SPAN & ESPAN & SPAN & ESPAN \\
\hline Sleep & $\mathbf{3 7 5}$ & $\mathbf{4 2 2}$ & 313 & $\mathbf{3 5 4}$ & $\mathbf{2 6 1}$ & 304 \\
\hline Idle & $\mathbf{1 2 4}$ & 77 & 186 & $\mathbf{1 4 5}$ & 238 & 195 \\
\hline
\end{tabular}

Table 1. Sleep and Idle time (in sec.) for $500 \mathrm{sec}$, simulation run

onds. From Table 1., it can be observed that E-SPAN consistently keeps the nodes in the Sleep mode more than the SPAN by around $13 \%$ of the time.

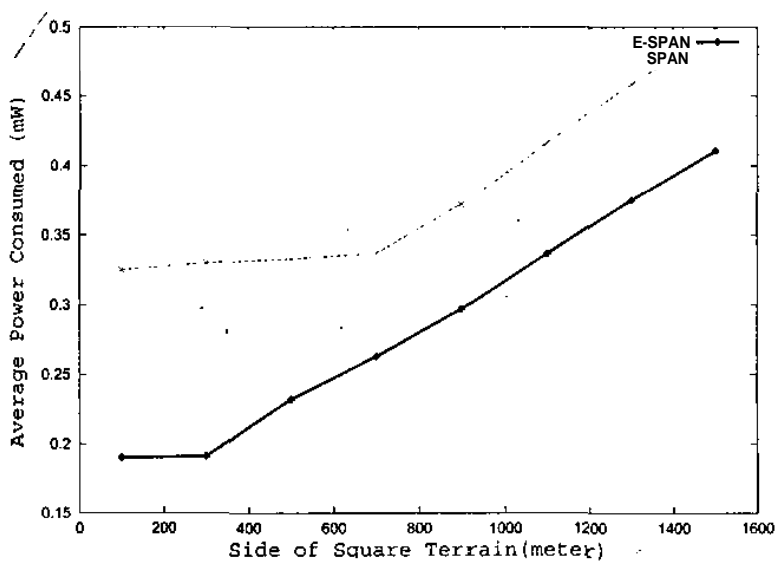

Figure 3. Average Power Consumed (in $\mathrm{mW}$ ) by each node vs. Side of Square Terrain

\section{Average Energy Consumptionfor varying Terruin Size}

Figure 3 shows the average power consumed (in $\mathrm{mW}$ ) by a node for varying terrain area. Our energy model assumes that network interface card spends $1400 \mathrm{~mW}$ in Send mode, $1000 \mathrm{~mW}$ in Receive mode, $830 \mathrm{~mW}$ in ldlemodeand $130 \mathrm{~mW}$ in Sleep mode $[1,4]$. The energy consumed during transmit and receive is a factor of packet size. Figure $\mathbf{3}$ shows that while using E-SPAN there is reduction in average power consumption. E-SPAN proves to be $20 \%$ more power efficient. This is in tune with the observations of the increase in Sleep time for E-SPAN as presented in Table 1.

During all our simulations we observed network capacity is not affected significantly with E-SPAN implementation.

\section{FUTURE WORK}

Our simulations show that due to the use of directional antenna E-SPAN is $20 \%$ more power efficient compared to SPAN. Considering the fact that the directional antenna gain used in this exercise was $7.7 \mathrm{~dB}$, one could technically argue that the reduction in energy should be of the order of 2.7 times energy gain at the best case. However the discrepancy in the improvement that we get can be accounted for by our observation during the simulations, that in E-SPAN there 


\section{Cellular Networks/679}

is a marginal increase in transmission failures. Further, the . present implementation of D-MAC is not optimal, while encountering hidden nodes. Our future efforts will be directed towards evolving better implementation of D-MAC, so that even in the presence of hidden nodes the energy saved in ESPAN will be close to the theoretical limit.

\section{REFERENCES}

[I] Chen Benjie, Jamieson Kyle, Balakrishnan Hand Morris Robert, "SPAN: An Energy-Efficient Coordination Algorithm for Topology Maintenance in Ad Hoc. Wireless Networks", ACM Wireless Networks Journal, Volume 8, Number 5, September, 2002.

[2] Choudhury R.R., Yang Xue, Ramanathan Ram, and Vaidya Nitin, "Using Directional Antennas for Medium Access Control in Ad Hoc Networks", $A C M$ International Conference on Mobile Computing and Networking (MobiCom), September 2002.

[3] Choudhury R.R. and Vaidya Nitin, "Ad Hoc Routing Using Directional,Antennas", Technical Report; August 2002.

[4] Feeney, L.M. and Nilsson, M., "Investigating the energy consumption of a wireless network interface in an ad hoc networking environment.", Proceedings of IEEE Infocom, Anchorage AK, April, 2001.

[5] Ram Ramanathan, "On the Performance of Ad Hoc Networks with Beamforming Antennas", Mobihoc 2001.

[6] Zhuochuan Huang, Chien-Chung Sben, "A Comparison Study of Omni-directional and Directional MAC Protocols for Ad hoc Networks", Pmceedings of IEEE Globecom 2002. Taipei, Taiwan, Nov2002.

[7] IEEE 802.1I, "Wireless LAN Medium Access Control and Physical Layer specifications", IEEE Computer Society: LAN MAN Standards Committee, (August 1999).

[8] Network Simulator ns-2, http://wwwisi eduilvint/nsnam.

[9] Puneet Sethi, Gautam Barua, "Dynamic Cluster Management in Ad hoc Networks", HPCAsia 2002. 